Nuclear Physics B320 (1989) 135-159

North-Holland, Amsterdam

\title{
THE SUPERMEMBRANE IS UNSTABLE
}

\author{
B. DE WIT \\ Institute for Theoretical Physics, University of Utrecht, Princetonplein 5 . \\ 3584 CC Utrecht, The Netherlands
}

M. LÜSCHER

Deutsches Elektronen-Synchroton, DFSY, Notkestrasse 85, 2000 Hamburg 52, FRG

H. NICOLAI

II Institute for Theoretical Physics, University of Hamburg, Luruper Chaussee 149, 2000 Hamburg 50, FRG

Received 21 November 1988

\begin{abstract}
We give a rigorous proof that the quantum mechanical hamiltonians of a class of supersymmetric matrix models have a continuous spectrum starting at zero. We are thus led to conclude that supermembranes (which can be regarded as a limit of such models) have a continuous mass spectrum and, in particular, no mass gap. This result can be understood as a manifestation of the instability of the supermembrane against deformations into long stringlike configurations. We also comment upon possible implications of this result for supersymmetric Yang-Mills theories.
\end{abstract}

\section{Introduction}

Supermembranes have been proposed as models of supersymmetric extended objects and alternative candidate theories of the fundamental interactions [1] (for reviews and further references see ref. [2]; earlier references on membrane theory are refs. $[3,4]$ ). It is the main purpose of this paper to demonstrate that the mass spectrum of these theories is continuous and extends down to zero. Although we cannot at present rule out the existence of discrete eigenvalues within the continuum or at its lower end, it is quite obvious that this result necessitates a revision of the commonly accepted physical interpretation of quantum supermembranes. Our analysis is based on a previous paper [5], where it was shown that the supermembrane is the large $N$ limit of a supersymmetric quantum mechanical model of $N \times N$ matrices, and within this regularization our arguments are completely rigorous.

The potential instability of (super-)membranes is already evident from classical considerations. In the light-cone gauge, the mass $\mathscr{M}$ of the bosonic membrane is 


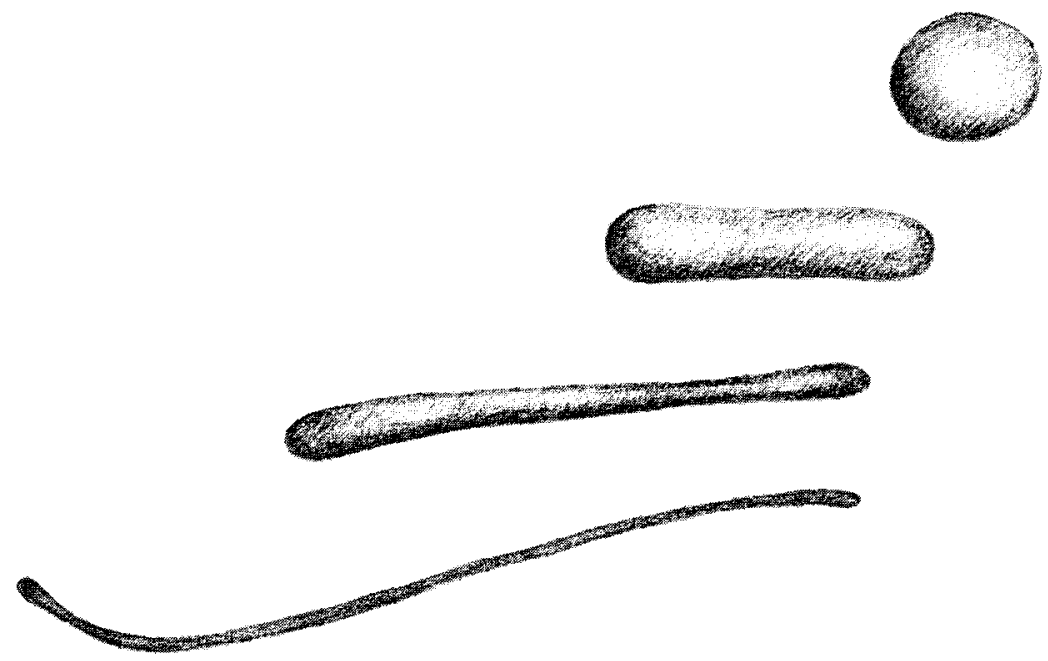

Fig. 1. Artist's view of an initially spherical membrane which moves along a valley of the potential (1.2) and ends up as a string. Contrary to the string model, such a degenerate membrane has, however, no energy proportional to its length associated with it, and it can therefore be arbitrarily long.

given by [4]

$$
\mathscr{M}^{2}=\int \mathrm{d}^{2} \sigma\left[P_{a}^{2}+V(X)\right]
$$

where

$$
V(X)=\frac{1}{2}\left(\epsilon^{r s} \partial_{r} X_{a} \partial_{s} X_{b}\right)^{2},
$$

and the center-of-mass momentum must, of course, be excluded from $P_{a}$ in eq. (1.1). The indices $a, b$ in these expressions label the transverse coordinates in target space and $r, s$ the spacelike membrane coordinates $\sigma=\left(\sigma_{1}, \sigma_{2}\right)$ (we will mostly adhere to the conventions of ref. [5] to which we refer the reader for further details). The potential (1.2) is non-negative, but it has valleys through which certain membrane coordinates can escape to infinity without increasing the mass. The membrane configurations at the bottom of these valleys are degenerate - the coordinates $X_{a}$ depend on only one of the membrane parameters - and a membrane moving out along the valley hence develops into a thinly stretched object whose mass can be made arbitrarily small (see fig. 1$)^{\star}$.

For the bosonic membrane this classical instability is cured by quantum mechanics (at least in the finite $N$ approximation): any wave function eventually gets stuck in the potential valleys because of its finite spread and because the valleys become increasingly narrow the further out one gets. Finite-energy wave functions consequently fall off rapidly and the spectrum of the quantum hamiltonian is purely

* It is amusing that Dirac's electron theory suffers from a related instability against quadrupolc deformations as was shown by P. Gnadig et al. [3]. 
discrete $[6,7]$. This rather welcome effect is, however, lost in the supersymmetric extension of the model, as we will show. Namely, the effective potential of the bosonic theory now gets additional contributions from the fermionic degrees of freedom which tend to cancel the bosonic ones. The wave functions then are no longer confined and the spectrum becomes continuous.

We emphasize that all higher $p$-branes suffer from such potential instabilities. To see this, one simply has to replace the potential (1.2) by the corresponding higher-dimensional metric determinant; again this determinant vanishes for configurations independent of one or more of the space-like $p$-brane coordinates. On the other hand, strings and superstrings have only one space-like coordinate and, apart from the center of mass motion, all modes are confined by the harmonic oscillator potential $\sum_{n=1}^{\infty} n X_{n}^{2}$.

To illustrate the remarks above, we will now discuss a simple quantum mechanical model that captures the physical essence of the argument while avoiding the technical complications of supermembranes. Consider the supersymmetric hamiltonian

$$
H=\frac{1}{2}\left\{Q, Q^{\dagger}\right\}
$$

where the supercharge is given by

$$
Q=Q^{\dagger}=\left(\begin{array}{cc}
-x y & i \partial_{x}+\partial_{y} \\
i \partial_{x}-\partial_{y} & x y
\end{array}\right) .
$$

These operators act on two-component wave functions $\psi(x, y), x$ and $y$ being the coordinates of a particle moving in the plane. Explicitly, the hamiltonian reads

$$
H=\left(\begin{array}{cc}
-\Delta+x^{2} y^{2} & x+i y \\
x-i y & -\Delta+x^{2} y^{2}
\end{array}\right)
$$

As in the supermembrane theory, we have $H \geqslant 0$, and the bosonic potential $x^{2} y^{2}$ does not increase in all directions but stays finite in a neighborhood of the coordinate axes. Thus, there are potential valleys along which, at the classical level, a particle can escape to infinity.

It has been known for some time that the associated bosonic hamilton operator $H_{\mathrm{B}}=-\Delta+x^{2} y^{2}$ nevertheless has a purely discrete spectrum [7]. One way to see this starts from the operator inequality

$$
H_{\mathrm{B}} \geqslant|x|
$$

which one obtains by regarding $H_{\mathrm{B}}$ as a harmonic oscillator in the variable $y$ at fixed $x$. The rhs of the inequality (1.6) is then simply the ground state energy of this 
oscillator. It follows from the inequality (1.6) that if one tries to move a wave packet to infinity along the potential valley $y=0$, the energy to be paid increases indefinitely irrespective of the shape of the wave packet. Since the bound (1.6) also applies when $x$ and $y$ are interchanged and hence $H_{\mathrm{B}} \geqslant(|x|+|y|) / 2$, it is clear that the particle is in fact confined and the spectrum of the bosonic hamiltonian is therefore discrete.

An interesting phenomenon now occurs, when we switch on the fermionic parts in the hamiltonian $H$. What happens is that the off-diagonal terms in eq. (1.5) can make a negative contribution to the total energy, and it is then possible to construct wave packets which can be moved to infinity along one of the potential valleys without paying an infinite amount of energy. In other words, whereas the system looked like a harmonic oscillator in the vicinity of the potential valleys before, it now looks like a supersymmetric harmonic oscillator, and since the ground state energy of this oscillator vanishes, it does not give rise to a confining force (the bound analogous to the inequality (1.6) becomes trivial).

To explicitly construct a wave packet which can be pushed to infinity, we make the ansatz

$$
\psi_{t}(x, y)=\chi(x-t) \varphi_{0}(x, y) \xi_{\mathrm{F}}
$$

where $t$ is a parameter, $\chi(x)$ a smooth function with compact support and

$$
\xi_{\mathrm{F}}=\frac{1}{\sqrt{2}}\left(\begin{array}{r}
1 \\
-1
\end{array}\right) \text {. }
$$

The scalar function $\varphi_{0}(x, y)$ will be determined later on. When $t$ is increased, the wave packet, eq. (1.7), is essentially translated in the $x$ direction so that, for $t \rightarrow \infty$, it moves along the potential valley $y=0$ to infinity. In particular, $\psi_{t}(x, y)$ vanishes unless $x$ is of order $t$.

The reason for the choice eq. (1.8) of the spinor $\xi_{\mathrm{F}}$ is that in this way the off-diagonal matrix elements in the hamilton operator make a maximal negative contribution to the energy of the wave packet, i.e. we have

$$
\xi_{\mathrm{F}}^{\mathrm{T}} H \xi_{\mathrm{F}}=H_{\mathrm{B}}-x
$$

Thus, the fermionic contribution to the expectation value of the energy in the state $\psi_{t}$ will be equal to $-t+\mathcal{O}(1)$ for large $t$. This is just enough to cancel the bosonic zero-point energy associated with the oscillations of the $y$-coordinate about the bottom of the potential valley (cf. the inequality (1.6)). We are thus led to choose

$$
\varphi_{0}(x, y)=\pi^{-1 / 4}|x|^{1 / 4} \exp \left(-\frac{1}{2}|x| y^{2}\right)
$$

which is the ground state wave function of the harmonic oscillator $-\partial_{y}^{2}+x^{2} y^{2}$. 
Now it is quite obvious that for $\nu=0,1,2$ we have

$$
\lim _{t \rightarrow \infty}\left(\psi_{t}, H^{\nu} \psi_{t}\right)=\int \mathrm{d} x \chi(x)^{*}\left(-\partial_{x}^{2}\right)^{\nu} \chi(x),
$$

and the wave packet so constructed can hence be shifted to infinity without having to supply an infinite amount of energy.

Eq. (1.11) also implies that the spectrum of $H$ is the whole interval $[0, \infty)$. Indeed, let $E \geqslant 0$ be any energy value and $\epsilon>0$. We can then choose $\chi(x)$ such that

$$
\|\chi\|=1, \quad\left\|\left(-\partial_{x}^{2}-E\right) \chi\right\|^{2}<\epsilon / 2
$$

where the norm \| $\|$ here refers to the usual norm for square integrable functions on the line. It follows that for large enough $t$, we have

$$
\left\|\psi_{t}\right\|=1, \quad\left\|(H-E) \psi_{t}\right\|^{2}<\epsilon,
$$

and since $\epsilon$ can be chosen arbitrarily small, this proves that $E$ is a spectral value of $H$ (see e.g. ref. [8]).

We mention in passing that an experimental realization of the model (1.5) would be an ideal focussing apparatus: when a wave packet moves out to infinity along a potential valley (which is the only way it can), the transverse width of the wave function is squeezed to zero (the expectation value of $y^{2}$ is proportional to $1 / t$ ). Because of the uncertainty relation, this would normally cost an infinite amount of energy, but here this is compensated by an equal amount of energy which comes from the orientation of the wave function in spin space. It would be amusing if an experimental set-up could be found which realizes the essential features of this little supersymmetric model.

To extend the simple ideas expounded above to the quantized supermembrane requires more work, and a large part of this paper will be devoted to rather technical details. For this reason, the reader should keep the above model in mind so as not to loose track of the thread of the argument. We emphasize at this point that the really essential properties of the system are the existence of potential valleys extending to infinity and supersymmetry. The latter is needed to guarantee the exact cancellation of the energy associated with the fluctuations of the bosonic variables transverse to the valley coordinates, and the energy coming from the orientation of the wave function in the fermion space.

In the light-cone gauge, the supermembrane hamiltonian can be written as

$$
H=\frac{1}{2} P_{a}^{A} P_{a A}+\frac{1}{4} f_{A B E} f_{C D}{ }^{E} X_{a}^{A} X_{b}^{B} X_{a}^{C} X_{b}^{D}-\frac{1}{2} i f_{A B C} X_{a}^{A} \theta^{B} \gamma^{a} \theta^{C}
$$

\footnotetext{
* The hamiltonian is related to the mass operator through $\mathscr{M}^{2}=2 H$. In what follows we use $H$ instead of $\mathscr{H}^{2}$ as the basic operator to be analyzed, because for the quantum mechanical matrix models introduced below, $H$ is really the hamilton operator in a standard notation.
} 
Supermembrane theories exist for spacetime dimensions $D=4,5,7$ and 11 [1], but for definiteness we will concentrate on the case $D=11$ which is also the most interesting in many respects (the extension of our proof of instability to the other cases is completely straightforward). The indices $a, b, \ldots$ thus run from 1 to 9 . Slightly deviating from the conventions of ref. [5], we here use real SO(9) spinors $\theta_{\alpha}^{A}$ with 16 components from the outset so that $\alpha, \beta, \ldots=1, \ldots, 16$. The corresponding $\gamma$-matrices obey $\left\{\gamma^{a}, \gamma^{b}\right\}=2 \delta^{a b}$. The indices $A, B, \ldots$ originate from a mode expansion of the target-space coordinates in terms of a complete set of functions $Y_{A}(\sigma)$, viz.

$$
X_{a}(\sigma)=\sum_{A} X_{a}^{A} Y_{A}(\sigma)
$$

The constants $f_{A B C}$ are then the structure constants of the group of area-preserving diffeomorphisms of the parameter manifold. For convenience, we shall assume throughout this paper that the basis $Y_{A}$ is real and orthonormal so that there is no distinction between upper and lower basis labels.

The group of area-preserving diffeomorphisms for spherical membranes [4] as well as for toroidal membranes [9] can be regarded as the limit of $\operatorname{SU}(N)$ for $N \rightarrow \infty$, and this suggests to regularize the theory by replacing this infinite-dimensional group by $\mathrm{SU}(N)$ [5]. In what follows we shall adopt this regularisation procedure, i.e. from now on the labels $A, B, \ldots$ run from 1 to $\operatorname{dim} G$ and $f_{A B C}$ denote the structure constants of $\mathrm{G}$, where $\mathrm{G}=\mathrm{SU}(N)$ will be referred to as the "gauge group" for reasons to become clear below. While the group $\operatorname{SU}(N)$ is the relevant choice in the context of the membrane theory, all our arguments below in fact remain valid for an arbitrary compact Lie group $G$ (of finite dimensionality). It is, however, important to appreciate that through the truncation of the number of degrees of freedom of the membrane, the hamiltonian (1.14) becomes mathematically well-defined. Furthermore, the supersymmetry of the model remains intact.

The canonical commutation relations of the basic variables are

$$
\begin{aligned}
& {\left[P_{a}^{A}, X_{b}^{B}\right]=-i \delta_{a b} \delta_{A B},} \\
& \left\{\theta_{\alpha}^{A}, \theta_{\beta}^{B}\right\}=\delta_{\alpha \beta} \delta_{A B} .
\end{aligned}
$$

To appreciate some of the technical complexities involved, the reader should realize that the dimensionality of the fermionic Fock space is $2^{8 \mathrm{dim} G}$. In particular, the 
spinor which will play a rôle similar to $\xi_{F}$ in the discussion above will have this many components.

The hamiltonian (1.14) is obviously invariant under adjoint transformations of $X_{a}^{A}$ and $\theta_{\alpha}^{A}$ by the elements of the gauge group $G$. In fact, the system looks exactly like a supersymmetric Yang-Mills gauge theory of constant gauge potentials and Fermi fields. As in this case, the wave functions of the system must satisfy Gauss' law, i.e. they must be gauge invariant (in the membrane theory this is a remnant of reparametrization invariance). The infinitesimal expression for this constraint reads

$$
L_{A} \psi=0 \text {, }
$$

where

$$
L_{A}=f_{A B C}\left(X_{a}^{B} P_{a}^{C}-\frac{1}{2} i \theta_{\alpha}^{B} \theta_{\alpha}^{C}\right)
$$

are the generators of the gauge transformations.

The main result of our paper is summarized by the following theorem.

Theorem. Let $\mathrm{G}$ be a compact Lie group (of finite dimensionality) and $H$ the associated Hamilton operator (1.14). Then, for any energy value $E \in[0, \infty)$ and any $\epsilon>0$, there exists a G-invariant wave function $\psi$ such that

$$
\|\psi\|=1 \quad \text { and } \quad\|(H-E) \psi\|^{2}<\epsilon
$$

In particular, the spectrum of $H$ is continuous and equal to the interval $[0, \infty)$.

As will become clear in the course of the proof of the theorem, the reason for the continuity of the spectrum of $H$ is that wave functions can escape to infinity along the directions corresponding to a Cartan subalgebra of the Lie algebra of G. In the context of the (regularised) supermembrane, this is thus exactly the instability alluded to above. In particular, for any finite $N$, the mass operator has a continuous spectrum and there is no gap between the zero mass states and the rest of the spectrum.

The organization of our paper is as follows. In section 2 we set up the necessary formalism. We define the Hilbert spaces of gauge-invariant and reduced wave functions, respectively, and explain how they are related. For simplicity, we shall work out the details only for the gauge group $\mathrm{G}=\mathrm{SU}(N)$, but the formulae are presented in such a way that their generalization to an arbitrary compact Lie group should be rather obvious. Section 3 will be devoted to the construction of the wave function $\psi$ and thus to the proof of the theorem above. In the concluding section we comment on the implications of our result. 


\section{Preliminaries}

\section{1. $\mathrm{SU}(N)$ NOTATIONS}

The Lie algebra $\mathrm{g}$ of the group $\mathrm{G}=\mathrm{SU}(N)$ consists of all complex $N \times N$ matrices $X$ with

$$
X^{\dagger}=-X, \quad \text { tr } X=0 .
$$

A G-invariant, positive definite scalar product on $\mathrm{g}$ is given by

$$
(X, Y)=-2 \operatorname{tr}(X Y)
$$

The set $\mathrm{k}$ of diagonal matrices $X \in \mathrm{g}$ is a maximal abelian subalgebra of $\mathrm{g}$ (also referred to as a Cartan subalgebra), and the corresponding subgroup of $\mathrm{G}$ will be denoted by $\mathrm{K}$.

The degrees of freedom parallel to $\mathrm{k}$ will later play the rôle of the valley coordinate $x$ in the discussion of section 1 , while the other degrees of freedom (i.e. those pointing in the direction of the orthogonal complement $k_{\perp}$ of $k$ ) will be confined by harmonic forces in the same way as the variable $y$. Having this in mind, we introduce a basis $T_{A}$ of $\mathrm{g}$ such that

$$
\begin{gathered}
\left(T_{A}, T_{B}\right)=\delta_{A B}, \\
T_{A} \in \mathrm{k} \quad \text { for } A=1, \ldots, N-1 .
\end{gathered}
$$

Accordingly, we shall adopt the convention that basis labels $i, j, \ldots$ are summed from 1 to $N-1$, while capital indices $I, J, \ldots$ from the middle of the alphabet run from $N$ to $N^{2}-1$. We finally note that any element $X$ of $\mathrm{g}$ can be written as $X=X^{A} T_{A}$ with real coefficients $X^{A}$, and that the structure constants

$$
f_{A B C}=\left(T_{A},\left[T_{B}, T_{C}\right]\right)
$$

are real and totally anti-symmetric in the indices $A, B, C$.

\subsection{THE HILBERT SPACE}

We here specify in concrete terms the space $\mathscr{H}$ of wave functions on which the hamiltonian (1.14) operates. To this end, we first introduce the fermion Fock space $\mathscr{H}_{\mathrm{F}}$. This is a linear space of dimension $2^{8 \mathrm{dimG}}$, which carries an irreducible representation of the Clifford algebra (1.17). As is well known, there is an essentially unique scalar product $(\xi, \eta)_{\mathrm{F}}$ on $\mathscr{H}_{\mathrm{F}}$ such that $\theta_{\alpha}^{A}$ is a hermitian operator for all $A$ and $\alpha$. 
$\mathscr{H}_{\mathrm{F}}$ also carries a unitary representation $V_{\mathrm{F}}(U), U \in \mathrm{G}$, of the gauge group $\mathrm{G}$. The infinitesimal generators

$$
L_{A}^{F}=\frac{1}{2 i} f_{A B C} \theta_{\alpha}^{B} \theta_{\alpha}^{C}
$$

of this representation satisfy the algebra

$$
\left[L_{A}^{F}, L_{B}^{F}\right]=i f_{A B C} L_{C}^{F} .
$$

By definition, they are bounded hermitian operators in $\mathscr{H}_{\mathrm{F}}$.

The Hilbert space $\mathscr{H}$ of physical states consists of wave functions

$$
\psi(X), \quad X=\left(X_{1}, \ldots, X_{9}\right), \quad X_{a} \in \mathrm{g},
$$

which take values in the fermion Fock space $\mathscr{H}_{\mathrm{F}}$. The scalar product in $\mathscr{H}$ is given by

$$
(\psi, \varphi)=\int \prod_{a, A} \mathrm{~d} X_{a}^{A}(\psi(X), \varphi(X))_{\mathrm{F}}
$$

Thus, $\mathscr{H}$ is the space of all measurable functions $\psi(X)$ which have finite norm and which, furthermore, are G-invariant, i.e. for all $U \in \mathrm{G}$ we have

$$
\psi\left(U X U^{-1}\right)=V_{\mathrm{F}}(U) \psi(X) .
$$

For differentiable wave functions this condition is equivalent to (1.18), if we choose the Schrödinger representation

$$
P_{a}^{A}=\frac{1}{i} \frac{\partial}{\partial X_{a}^{A}}
$$

for the momentum operators.

The hamilton operator (1.14) is initially defined as a differential operator on rapidly decaying smooth wave functions $\psi(X)$ and then extends to a self-adjoint operator in $\mathscr{H}$ through closure (cf. ref. [8], theorem X.40). It is known that there exist conserved supercharge operators $Q_{\alpha}$ acting in $\mathscr{H}$ such that

$$
\begin{gathered}
Q_{\alpha}^{\dagger}=Q_{\alpha}, \\
\left\{Q_{\alpha}, Q_{\beta}\right\}=2 \delta_{\alpha \beta} H .
\end{gathered}
$$

From this result one deduces that the hamiltonian (1.14) is non-negative. Although 
supersymmetry is crucial for our arguments, we will not need the explicit expressions for the supercharge operators.

\subsection{GAUGE FIXING}

The gauge invariance of the wave functions in the Hilbert space of states implies that they do not depend on a subset of variables. For the construction of wave packets that can be moved to infinity along one of the potential valleys, we have found that the presence of these redundant variables leads to some technical problems. We have therefore decided to eliminate most of them by fixing the gauge. In the way this is done here, the procedure is completely rigorous and does not in any way imply a mutilation of the original system.

The basic idea is that through a group transformation we can always diagonalize the matrix $X_{9}$, and a gauge invariant wave function $\psi(X)$ is hence completely determined when its values are known for all $X$ with $X_{9}$ diagonal. To work out this idea in detail, we need to introduce some further notations.

First, let $\mathrm{p}$ be the set of all matrices $Z$ of the form

$$
Z=i\left(\begin{array}{cccc}
\lambda_{1} & 0 & \ldots & 0 \\
0 & \lambda_{2} & \ldots & 0 \\
\vdots & \vdots & \ddots & \vdots \\
0 & 0 & \ldots & \lambda_{N}
\end{array}\right)
$$

where $\lambda_{n} \in \mathrm{R}$ and

$$
\sum_{n=1}^{N} \lambda_{n}=0, \quad \lambda_{1} \geqslant \lambda_{2} \geqslant \ldots \geqslant \lambda_{N} .
$$

Obviously, $\mathrm{p}$ is a subset of the Cartan subalgebra $\mathrm{k}$ whose interior $\dot{\mathrm{p}}$ is an open cone ( $\dot{p}$ is a "Weyl chamber"). The essential property of $\mathrm{p}$ is that for any given element $X$ of the Lie algebra g, there exists a unique $Z \in \mathrm{p}$ such that

$$
X=U Z U^{-1} \quad \text { for some } U \in \mathrm{G} \text {. }
$$

Furthermore, if all the eigenvalues of $X$ are pairwise different (in which case $X$ is referred to as a regular element), not only $Z$ is uniquely determined, but one can also show that the transformation $U$ is unique up to multiplications by an arbitrary element of the Cartan subgroup $\mathrm{K}$ from the right. In other words, eq. (2.15) defines a parametrization of $\mathrm{g}$ in terms of $Z \in \mathrm{p}$ and $U \in \mathrm{G} / \mathrm{K}$.

It is clear from the above that if we have some (complex valued) integrable function $f(X), X \in \mathrm{g}$, which is invariant under the action of the gauge group $\mathrm{G}$, a 
relation of the form

$$
\int \prod_{A} \mathrm{~d} X^{A} f(X)=C \int_{\mathrm{p}} \prod_{i} \mathrm{~d} Z^{i} \Delta(Z) f(Z)
$$

must hold, where $C$ is a positive constant and $\Delta(Z)$ the appropriate Faddeev-Popov determinant. There are various ways to compute $\Delta(Z)$. For example, introducing coordinates on $\mathrm{G} / \mathrm{K}$ and using the representation (2.15), one obtains a (non-linear) parametrization of $\mathrm{g}$. For symmetry reasons, the associated jacobian is a product of two factors, one depending on $Z$ and the other on $U$. Since $f(X)$ is independent of $U$, the integration over $\mathrm{G} / \mathrm{K}$ is hence trivial and gives the constant $C$ which is essentially the volume of $\mathrm{G} / \mathrm{K}$. The result of this calculation (which has been known for a long time [10]) is

$$
\Delta(Z)=\operatorname{det} z
$$

where $z_{I J}$ is the real anti-symmetric matrix defined through

$$
\left[Z, T_{I}\right]=z_{I J} T_{J}
$$

More explicitly, we have

$$
z_{I J}=Z^{k} f_{k J}
$$

and the eigenvalues of $z_{I J}$ are just the (non-zero) roots of the Lie algebra g. Thus, for $\mathrm{G}=\mathrm{SU}(N)$ one finds

$$
\operatorname{det} z=\prod_{m<n}^{N}\left(\lambda_{m}-\lambda_{n}\right)^{2}
$$

Note that $\operatorname{det} z$ vanishes when some of the eigenvalues of $Z$ coincide, i.e. at the boundary of the Weyl chamber $p$. Whenever this happens, the matrix $Z$ becomes degenerate in the sense that a linear combination of the generators $T_{I}$ commutes with it, and the matrix $z_{I J}$ has null vectors.

In passing we mention that from the representation (2.19) and the general properties of the structure constants it follows that the pfaffian of $z_{I J}$ satisfies the equation

$$
\frac{\partial}{\partial Z^{i}} \frac{\partial}{\partial Z^{i}}(\operatorname{det} z)^{1 / 2}=0
$$

a result that will be useful later on.

After these lengthy preparations, we are now in a position to explain the gauge fixing procedure. Suppose $\psi$ is any element of the Hilbert space $\mathscr{H}$ of states. We 
then define an associated reduced wave function $\hat{\psi}$ through

$$
\hat{\psi}\left(X_{1}, \ldots, X_{8}, Z\right)=\left.(C \operatorname{det} z)^{1 / 2} \psi(X)\right|_{X_{9}=Z},
$$

where $Z \in \mathrm{p}$ and the constant $C$ is the same as in eq. (2.16). It should be quite clear at this point that the reduced wave function $\hat{\psi}$ contains no less information than $\psi$ itself. In fact, recalling eq. (2.10), one quickly verifies that

$$
\psi(X)=(C \operatorname{det} z)^{-1 / 2} V_{\mathrm{F}}(U) \hat{\psi}\left(U^{-1} X_{1} U, \ldots, U^{-1} X_{8} U, Z\right),
$$

where $U \in \mathrm{G}$ and $Z \in \mathrm{p}$ are such that $X_{9}=U Z U^{-1}$.

In what follows, the aim is to rewrite everything in terms of reduced wave functions. First, we define $\hat{\mathscr{H}}$ to be the Hilbert space of wave functions $\hat{\psi}\left(X_{1}, \ldots, X_{8}, Z\right), X_{a} \in \mathrm{g}, Z \in \mathrm{p}$, with values in $\mathscr{H}_{\mathrm{F}}$, which are normalizable with respect to the scalar product

$$
(\hat{\psi}, \hat{\varphi})=\int \prod_{a, A} \mathrm{~d} X_{a}^{A} \int_{\mathrm{p}} \prod_{i} \mathrm{~d} Z^{i}(\hat{\psi}(X, Z), \hat{\varphi}(X, Z))_{\mathrm{F}}
$$

and which are invariant under the action of the Cartan subgroup $\mathrm{K}$, i.e. for all $U \in \mathrm{K}$ we have

$$
\hat{\psi}\left(U X U^{-1}, Z\right)=V_{\mathrm{F}}(U) \hat{\psi}(X, Z) .
$$

It follows from the definition (2.22) and the integration rule (2.16) that the reduced wave function $\hat{\psi}$ associated to any $\psi \in \mathscr{H}$ is an element of $\hat{\mathscr{H}}$ and, furthermore, that

$$
(\hat{\psi}, \hat{\psi})=(\psi, \psi)
$$

In other words, the mapping $\psi \mapsto \hat{\psi}$ is unitary.

A crucial observation now is that if $\hat{\psi}$ is an arbitrary element of $\hat{\mathscr{H}}$, there exists a unique wave function $\psi \in \mathscr{H}$ such that $\hat{\psi}$ is the reduced wave function belonging to $\psi$, i.e. such that eq. (2.22) holds. This implies, for example, that later on when we construct a wave packet analogous to $\psi_{t}$ in section 1 , it is sufficient to specify a wave function $\hat{\psi}_{t}(X, Z)$ and to check that it is normalizable and $\mathrm{K}$-invariant.

At this point we would like to caution the reader that, although the reconstruction of a full wave function $\psi$ from a reduced wave function $\hat{\psi}$ always yields an element of $\mathscr{H}$, one does not always get a smooth function when $\hat{\psi}$ is differentiable. The reason for this is that the representation of $X_{9}$ in terms of $U \in \mathrm{G} / \mathrm{K}$ and $Z \in \mathrm{p}$ is only differentiable when $Z$ is in the interior of $\mathrm{p}$, i.e. when $X_{9}$ is regular. Fortunately, we do not have to study this problem any further, because later on we shall only encounter differentiable wave functions $\hat{\psi}\left(X_{1}, \ldots, X_{8}, Z\right)$, which vanish 
unless $Z$ is in a certain compact subset of $\dot{\mathrm{p}}$. Thus, the singular boundary points of $\mathrm{p}$ are avoided and $\hat{\psi}$ hence corresponds to a differentiable full wave function $\psi$.

Our next task is to evaluate the hamiltonian (1.14) for the reduced wave functions. This reduced hamiltonian, which we will denote by $\hat{H}$, is defined by

$$
\hat{H} \hat{\psi}=\widehat{H \psi} .
$$

Obviously, one has

$$
\left(\hat{\psi}, \hat{H}^{v} \hat{\psi}\right)=\left(\psi, H^{v} \psi\right)
$$

for any arbitrary power $\nu$.

The non-trivial part in the computation of $\hat{H}$ concerns the evaluation of the terms coming from $\left(P_{9}^{A}\right)^{2}$. Before doing this let us define the matrix $w_{I J}$, which is the inverse of the matrix $z_{I J}$, viz.

$$
w_{I J} z_{J K}=\delta_{I K} .
$$

It is not hard to prove that

$$
w_{I J} f_{I J K}=0 .
$$

Furthermore, we introduce the part of the G-generators that pertains to $X_{9}^{A}$ through

$$
L_{A}^{9}=-i f_{A B C} X^{B} \frac{\partial}{\partial X^{C}}
$$

where here and henceforth we will suppress the index 9 , so that $X^{A} \equiv X_{9}^{A}$ and the indices $a$ will only run from 1 to 8 . With these notations it is easy to show that for $Z \in \mathrm{p}$ and any function $f$ of $X$ one has

$$
\left.\frac{\partial}{\partial X^{I}} f(X)\right|_{X=Z}=-\left.i w_{I J} L_{J}^{9} f(X)\right|_{X=Z}
$$

where $X$ is set equal to $Z$ only after all derivatives are performed. Applying this result to the function $\partial f(X) / \partial X^{I}$, using the commutation relation for $L_{J}^{9}$ and $\partial / \partial X^{I}$, eq. (2.30) and again eq. (2.32), one straightforwardly finds

$$
\left.\frac{\partial}{\partial X^{I}} \frac{\partial}{\partial X^{I}} f(X)\right|_{X=Z}=-\left.\left(\left(w^{\mathrm{T}} w\right)_{I J} L_{I}^{9} L_{J}^{9}+w_{I J} f_{I J k} \frac{\partial}{\partial X^{k}}\right) f(X)\right|_{X=Z} .
$$

If we now apply this identity to a wave function $\psi \in \mathscr{H}$, the constraint (1.18) can be invoked to replace the operator $L_{I}^{9}$ on the rhs by the operator $\hat{L}_{I}$ defined by

$$
\hat{L}_{I} \equiv L_{I}-L_{I}^{9} \text {. }
$$


The advantage of this replacement is that $\hat{L}_{I}$ does not involve the variables $X^{A}$ and is therefore not affected by the substitution $X=Z$. To sum up, the contribution of $P_{9}^{A} P_{9}^{A}$ to the reduced hamilton operator is (leaving out an overall factor of $\frac{1}{2}$ )

$$
\left(w^{\mathrm{T}} w\right)_{I J} \hat{L}_{I} \hat{L}_{J}-\sqrt{\operatorname{det} z}\left(\frac{\partial}{\partial Z^{k}}-w_{I J} f_{I J k}\right) \frac{\partial}{\partial Z^{k}} \frac{1}{\sqrt{\operatorname{det} z}} .
$$

Finally, we note that the second term in this formula is actually equal to $-\left(\partial / \partial Z^{k}\right)^{2}$, as one may show by using

$$
\frac{\partial}{\partial Z^{k}} \ln \operatorname{det} z=-w_{I J} f_{I J k}
$$

and eq. (2.21).

After these manipulations we can now give the reduced hamiltonian $\hat{H}$. Before doing so we decompose the coordinates $X_{a}^{A}$ into $Z_{a}^{i} \equiv X_{a}^{i}$ and $Y_{a}^{I} \equiv X_{a}^{I}$, i.e.

$$
X_{a}^{A} \rightarrow\left(Z_{a}^{i}, Y_{a}^{I}\right), \quad a=1, \ldots, 8
$$

The hamiltonian $\hat{H}$ is then divided into four terms,

$$
\hat{H}=H_{1}+H_{2}+H_{3}+H_{4},
$$

which are separately invariant under the Cartan subgroup $\mathrm{K}$. They are defined by

$$
\begin{aligned}
H_{1}= & -\frac{1}{2}\left(\frac{\partial}{\partial Z^{k}}\right)^{2}-\frac{1}{2}\left(\frac{\partial}{\partial Z_{a}^{k}}\right)^{2}, \\
H_{2}= & -\frac{1}{2}\left(\frac{\partial}{\partial Y_{a}^{I}}\right)^{2}+\frac{1}{2}\left(z^{\mathrm{T}} z\right)_{I J} Y_{a}^{I} Y_{a}^{J}, \\
H_{3}= & -\frac{1}{2} i \theta^{I}\left(z_{I J} \gamma_{9}+z_{I J}^{a} \gamma_{a}\right) \theta^{J}, \\
H_{4}= & \frac{1}{4} f_{A I J} f_{A K L} Y_{a}^{I} Y_{b}^{J} Y_{a}^{K} Y_{b}^{L}+f_{A I J} f_{A K L} Z_{a}^{i} Y_{b}^{J} Y_{a}^{K} Y_{b}^{L} \\
& +\frac{1}{2} f_{A i J} f_{A K L} Z_{a}^{i} Y_{b}^{J}\left(Z_{a}^{k} Y_{b}^{L}-Z_{b}^{k} Y_{a}^{L}\right) \\
& +\frac{1}{2}\left(w^{\mathrm{T}} w\right)_{I J} \hat{L}_{I} \hat{L}_{J}-\frac{1}{2} i f_{I A B} Y_{a}^{I} \theta^{A} \gamma_{a} \theta^{B},
\end{aligned}
$$

where we have used the notation

$$
z_{I J}^{a}=Z_{a}^{k} f_{k I J}
$$




\section{The spectrum of $H$}

In this section, we prove the theorem stated in section 1, following in outline the argumentation we have applied there to the simple operator (1.5).

\subsection{ANSATZ FOR THE TRIAL WAVE FUNCTION $\psi$,}

The wave packet to be constructed will be moved to infinity along the potential valley characterized by $X_{a} \in \mathrm{k}$. More precisely, using reduced wave functions, we choose the variables $Z^{i}$ to play the rôle of the variable $x$ in the model studied in section 1 . Thus, we introduce a smooth function $\chi\left(Z, Z_{a}\right), Z, Z_{a} \in \mathbf{k}$, with compact support, which will appear in the trial wave function $\hat{\psi}_{t}$ in the form $\chi\left(Z-t V, Z_{a}\right)$. where $V$ is a constant diagonal matrix given by

$$
V=i\left(\begin{array}{cccc}
s-1 & 0 & \ldots & 0 \\
0 & s-2 & \cdots & 0 \\
\vdots & \vdots & \ddots & \vdots \\
0 & 0 & \cdots & s-N
\end{array}\right), \quad s=(N+1) / 2
$$

When $t$ is increased the wave packet will thus be translated along the valley in the direction specified by eq. (3.1). Because $\chi$ has compact support, this implies that differences between the eigenvalues $\lambda_{m}$ of $Z$ satisfy

$$
\lambda_{m}-\lambda_{n}=(n-m) t+\mathcal{O}(1)
$$

for $Z$ in the support of $\hat{\psi}_{t}$. In particular, for large enough $t, \hat{\psi}_{t}$ is supported in the Weyl chamber $p$, as appropriate for reduced wave functions.

The complete expression for the wave packet $\hat{\psi}_{t}$ reads

$$
\hat{\psi}_{t}\left(Z, Z_{a}, Y_{a}^{I}\right)=\chi\left(Z-t V, Z_{a}\right) \varphi_{0}\left(Z, Y_{a}^{I}\right) \xi_{\mathrm{F}}\left(Z, Z_{a}\right),
$$

where $\varphi_{0}$ and $\xi_{\mathrm{F}}$ are the ground-state wave functions of the hamiltonians $H_{2}$ and $H_{3}$, respectively. The notation has been chosen in such a way that the analogy with the wave function (1.7) is completely evident. Furthermore, as we shall show later on, the ground-state wave functions of the hamiltonians $\mathrm{H}_{2}$ and $\mathrm{H}_{3}$ are separately invariant under $\mathrm{K}$, while $\chi$ depends only on $\mathrm{K}$-invariant variables. Consequently, $\hat{\psi}_{t}$ is $\mathrm{K}$-invariant as well and hence an element of the Hilbert space $\hat{\mathscr{H}}$ of reduced wave functions. Furthermore, for sufficiently large $t, \hat{\psi}_{t}$ vanishes unless $Z$ is contained in a compact subset of the interior of $\mathrm{p}$ so that the associated full wave function $\psi_{t}$ is smooth (as we have discussed in section 2).

We now proceed to discuss the wave functions $\varphi_{0}$ and $\xi_{\mathrm{F}}$ in detail. 


\subsection{THE GROUND STATE OF $\mathrm{H}_{2}$}

Consider the positive-definite symmetric matrix

$$
\Omega=\sqrt{z^{\mathrm{T}} z}
$$

which has two-fold degenerate eigenvalues $\lambda_{m}-\lambda_{n}(m<n)$, so that

$$
\operatorname{det} \Omega=\prod_{m<n}^{N}\left(\lambda_{m}-\lambda_{n}\right)^{2}, \quad \operatorname{tr} \Omega=2 \sum_{m<n}^{N}\left(\lambda_{m}-\lambda_{n}\right)
$$

For fixed $Z$, the hamiltonian $H_{2}$ describes a set of $8\left(N^{2}-N\right)$ harmonic oscillators with frequencies $\lambda_{m}-\lambda_{n}$. The wave function for its ground state is therefore easy to write down, and reads as follows

$$
\varphi_{0}\left(Z, Y_{a}^{I}\right)=\pi^{2\left(N-N^{2}\right)}(\operatorname{det} \Omega)^{2} \exp \left(-\frac{1}{2} \Omega_{I J} Y_{a}^{I} Y_{a}^{J}\right)
$$

The ground-state energy is given by

$$
H_{2} \varphi_{0}=4 \operatorname{tr} \Omega \varphi_{0}
$$

Furthermore, $\varphi_{0}$ is normalized such that

$$
\left(\varphi_{0}, \varphi_{0}\right)_{Y}=1
$$

where the norm in eq. (3.8) involves only an integration over the coordinates $Y_{a}^{I}$, viz.

$$
(\varphi, \psi)_{Y} \equiv \int \prod_{a, I} \mathrm{~d} Y_{a}^{\prime} \varphi^{*} \psi
$$

Observe that the ground-state wave function (3.6) is manifestly $\mathrm{K}$-invariant, because the matrix $\Omega_{I J}$ commutes with the rotation matrices representing the action of $\mathrm{K}$ on $\mathrm{k}_{\perp}$.

When $Z$ becomes large in the way we have described above, the potential valley will become more and more narrow, so that expectation values of the transverse coordinates $Y_{a}^{I}$ will be suppressed. More precisely, if $P(Y)$ is a homogeneous polynomial of degree $\delta$, we have

$$
\left|\left(\varphi_{0}, P(Y) \varphi_{0}\right)_{Y}\right| \leqslant C t^{-\delta / 2}
$$

This result may be extended to (homogeneous) operators $\mathscr{D}_{1}, \mathscr{D}_{2}, \ldots$, which may contain derivatives with respect to $Y_{a}^{I}$ or $\lambda_{m}$ with polynomial coefficients. We can 
assign a dimension to such operators by defining

$$
\begin{array}{cc}
\operatorname{dim} \lambda_{m}=1, & \operatorname{dim} Y_{a}^{l}=-\frac{1}{2}, \\
\operatorname{dim} \partial / \partial \lambda_{m}=-1, & \operatorname{dim} \partial / \partial Y_{a}^{I}=\frac{1}{2} .
\end{array}
$$

It is then straightforward to show that

$$
\left|\left(\mathscr{D}_{1} \varphi_{0}, \mathscr{D}_{2} \varphi_{0}\right)_{Y}\right| \leqslant C t^{\operatorname{dim} \mathscr{D}_{1}+\operatorname{dim} \mathscr{D}_{2}}
$$

for suitable constants $C$ and sufficiently large $t$.

\subsection{THE GROUND STATE OF $H_{3}$}

From rather general arguments one can show that, for fixed $Z$ and $Z_{a}$, the ground-state energy of $\mathrm{H}_{3}$ is given by

$$
H_{3} \xi_{\mathrm{F}}=-8 \sum_{m<n}^{N} \omega_{m n} \xi_{\mathrm{F}},
$$

where $\omega_{m n}$ is defined in terms of the eigenvalues $\lambda_{m}$ and $\lambda_{m}^{a}$ of $Z$ and $Z_{a}$ by

$$
\omega_{m n}=\sqrt{\left(\lambda_{m}-\lambda_{n}\right)^{2}+\sqrt{\left(\lambda_{m}^{a}-\lambda_{n}^{a}\right)^{2}}}
$$

However, to determine the wave function $\xi_{\mathrm{F}}$ explicitly requires more work. Let us start by diagonalizing the (commuting) matrices $z_{I J}$ and $z_{I J}^{a}$. Their eigenvectors are precisely the (complex) root vectors ${ }^{\star} E_{m n}^{I}(m \neq n)$, satisfying

$$
\begin{aligned}
& z_{I J} E_{m n}^{J}=i\left(\lambda_{m}-\lambda_{n}\right) E_{m n}^{I}, \\
& z_{I J}^{a} E_{m n}^{J}=i\left(\lambda_{m}^{a}-\lambda_{n}^{a}\right) E_{m n}^{I}, \\
& \left(E_{m n}^{I}\right)^{*}=E_{n m}^{I} .
\end{aligned}
$$

These vectors define a complete orthonormal set, i.e.,

$$
\begin{aligned}
\sum_{I}\left(E_{m n}^{I}\right)^{*} E_{p q}^{I} & =\delta_{m p} \delta_{n q} \quad(m \neq n, p \neq q), \\
\sum_{m \neq n}\left(E_{m n}^{I}\right)^{*} E_{m n}^{J} & =\delta_{I J} .
\end{aligned}
$$

\footnotetext{
* Not to be confused with the roots, which are $(N-1)$-component vectors, whereas the $E_{m n}$ 's have $N^{2}-N$ components.
} 
Expanding $\theta^{I}$ in terms of the vectors $E_{m n}^{I}$,

$$
\theta^{I}=\sum_{m \neq n} \theta^{m n} E_{m n}^{I}
$$

we have the reality property

$$
\theta^{m n \dot{\dagger}}=\theta^{n m},
$$

and the anticommutation relations

$$
\left\{\theta_{\alpha}^{m n}, \theta_{\beta}^{p q}\right\}=\delta_{\alpha \beta} \delta^{m q} \delta^{n p} .
$$

Notice that by rewriting the real spinors $\theta^{I}$ in a complex basis we have now a natural split into $\frac{1}{2}\left(N^{2}-N\right)$ creation and $\frac{1}{2}\left(N^{2}-N\right)$ annihilation operators. In terms of $\theta^{m n}, H_{3}$ takes the form

$$
H_{3}=\sum_{m<n}^{N} \theta^{m n \dagger}\left[\left(\lambda_{m}-\lambda_{n}\right) \gamma_{9}+\left(\lambda_{m}^{a}-\lambda_{n}^{a}\right) \gamma_{a}\right] \theta^{m n}
$$

We now redefine the spinors by a $\lambda$-dependent $\operatorname{Spin}(9)$ transformation,

$$
\tilde{\theta}^{m n}=\frac{1}{\sqrt{2 \omega_{m n}}}\left(\sqrt{\omega_{m n}+\lambda_{m}-\lambda_{n}}-\frac{\left(\lambda_{m}^{a}-\lambda_{n}^{a}\right) \gamma_{a} \gamma_{9}}{\sqrt{\omega_{m n}+\lambda_{m}-\lambda_{n}}}\right) \theta^{m n} \quad(m<n),
$$

so that the $\tilde{\theta}^{m n}$ and $\tilde{\theta}^{m n \dagger}$ satisfy the same anticommutation relations as $\theta^{m n}$ and $\theta^{m n \dagger}$, although they no longer commute with the bosonic momentum operators. The hamiltonian $\mathrm{H}_{3}$ can then be written as

$$
\begin{aligned}
H_{3} & =\sum_{m<n}^{N} \omega_{m n} \tilde{\theta}^{m n \dagger} \gamma_{9} \tilde{\theta}^{m n} \\
& =\sum_{m<n}^{N} \omega_{m n}\left(\tilde{\theta}_{+}^{m n \dagger} \tilde{\theta}_{+}^{m n}+\tilde{\theta}_{-}^{m n} \tilde{\theta}_{-}^{m n \dagger}-8\right),
\end{aligned}
$$

where we have used the anticommutation relations to split off the first two terms which are positive definite. Note that we have employed "chiral" spinors in eq. (3.22), defined by the projections

$$
\theta_{ \pm} \equiv \frac{1 \pm \gamma_{9}}{2} \theta
$$

which have only eight non-vanishing components labelled by $\alpha^{\prime}$. 
The ground-state wave function can now be written as

$$
\xi_{\mathrm{F}}=\left(\prod_{m<n}^{N} \prod_{\alpha^{\prime}=1}^{8}\left(\tilde{\theta}_{-}^{m n \dagger}\right)_{\alpha^{\prime}}\right) \xi_{0}
$$

where the Fock vacuum $\xi_{0}$ satisfies

$$
\theta^{m n} \xi_{0}=0 \text { for all } m<n
$$

and is chosen $\lambda$-independent and normalized to $1^{\star}$. The only $\lambda$-dependence in $\xi_{\mathrm{F}}$ therefore comes from the operator multiplying $\xi_{0}$ in eq. (3.24). Furthermore, $\xi_{\mathrm{F}}$ is also normalized, i.e. we have

$$
\left(\xi_{\mathrm{F}}, \xi_{\mathrm{F}}\right)_{\mathrm{F}}=1
$$

Note that the condition (3.25) does not completely determine the fermionic Fock vacuum, because it does not specify how the remaining fermionic operators $\theta^{i}$ act on it. However, further specification of $\xi_{\mathrm{F}}$ is unnecessary, because $\theta^{i}$ only occurs in $\mathrm{H}_{4}$ and this term makes a negligible contribution to the energy of the wave packet $\hat{\psi}_{i}$, as we shall show below.

To verify the consistency of our ansatz (3.24) we must also check the K-invariance of $\xi_{F}$ which is not immediately obvious. To do so we recall that the generators which must annihilate $\xi_{\mathrm{F}}$ are given by (cf. eq. (2.6))

$$
L_{k}^{\mathrm{F}}=\frac{1}{2 i} f_{k I J} \theta_{\alpha}^{I} \theta_{\alpha}^{J}
$$

Using the expansion (3.17) and the decomposition of the $\theta^{m n}$ 's into creation and annihilation operators, one finds that a sufficient condition for $\xi_{\mathrm{F}}$ to be K-invariant is

$$
\left(\theta_{\alpha}^{m n} \theta_{\alpha}^{m n^{\dagger}}-\theta_{\alpha}^{m n^{\dagger}} \theta_{\alpha}^{m n}\right) \xi_{\mathrm{F}}=0 \quad \text { for all } m<n
$$

Since the Spin(9) transformation (3.21) is unitary and does not mix the $\mathrm{SU}(N)$ indices, eq. (3.28) is equivalent to

$$
\left(\tilde{\theta}_{\alpha}^{m n} \tilde{\theta}_{\alpha}^{m n \dagger}-\tilde{\theta}_{\alpha}^{m n \dagger} \tilde{\theta}_{\alpha}^{m n}\right) \xi_{\mathrm{F}}=0 \quad \text { for all } m<n
$$

From eqs. (3.24) and (3.25), it is now straightforward to see that $\xi_{\mathrm{F}}$ does indeed satisfy this requirement.

« To show that eq. (3.24) is the ground state of $H_{3}$, observe that the Spin(9) rotation (3.21) does not mix the $\operatorname{SU}(N)$ indices, so that we also have $\tilde{\theta}^{m n} \xi_{0}=0$ for $m<n$. 
For large $t$ the operators $\tilde{\theta}^{m n}$ tend to $\theta^{m n}$, so that $\xi_{\mathrm{F}}$ becomes independent of $\lambda$, viz.

$$
\xi_{\mathrm{F}}=\left(\prod_{m<n}^{N} \prod_{\alpha^{\prime}=1}^{8}\left(\theta_{-}^{m n^{\dagger}}\right)_{\alpha^{\prime}}\right) \xi_{0}+\mathcal{O}\left(\frac{1}{t}\right) .
$$

As a result (multiple) derivations $\mathscr{D}$ with respect to the eigenvalues $\lambda_{m}$ and $\lambda_{m}^{a}$ acting on $\xi_{\mathrm{F}}$ will satisfy

$$
\left\|\mathscr{D} \xi_{\mathrm{F}}\right\|_{\mathrm{F}} \leqslant \frac{C}{t}
$$

for large $t$.

\subsection{PROOF OF THE THEOREM}

We will now come to the central result of this paper. First we observe that since the ground-state wave functions $\varphi_{0}$ and $\xi_{\mathrm{F}}$ are normalized, the norm of the trial wave functions $\psi_{t}$ is given by

$$
\left(\hat{\psi}_{t}, \hat{\psi}_{t}\right)=\langle\chi, \chi\rangle
$$

where the scalar product $\langle$,$\rangle is defined by$

$$
\left\langle\chi_{1}, \chi_{2}\right\rangle=\int \prod_{i} \mathrm{~d} Z^{i} \prod_{k, a} \mathrm{~d} Z_{a}^{k} \chi_{1}^{*} \chi_{2}
$$

Next, we note that

$$
\left(H_{2}+H_{3}\right) \hat{\psi}_{t}=8 \sum_{m<n}^{N}\left(\lambda_{m}-\lambda_{n}-\omega_{m n}\right) \hat{\psi}_{t}
$$

When $\lambda_{m}-\lambda_{n} \rightarrow \infty$, the rhs in this equation will go to zero, which can be understood from the fact that the hamiltonian $\mathrm{H}_{2}+\mathrm{H}_{3}$ describes a set of supersymmetric harmonic oscillators in that limit. Using that $\chi$ is of compact support, we conclude that

$$
\lim _{t \rightarrow \infty}\left\|\left(H_{2}+H_{3}\right) \hat{\psi}_{t}\right\|=0
$$

Furthermore, one can easily establish that the operator $H_{4}$ is a sum of terms of dimension $\delta \leqslant-\frac{1}{2}$ in the sense of (3.11). Using the inequalities (3.12) and (3.30), it follows that

$$
\lim _{t \rightarrow \infty}\left\|H_{4} \hat{\psi}_{t}\right\|=0
$$


so that altogether we nave

$$
\lim _{t \rightarrow \infty}\left(\psi_{t}, H^{\nu} \psi_{t}\right)=\lim _{t \rightarrow \infty}\left(\hat{\psi}_{t}, H_{1}^{\nu} \hat{\psi}_{t}\right)
$$

for $\nu=1,2$.

If the derivatives contained in $H_{1}$ act on $\varphi_{0}$ or $\xi_{\mathrm{F}}$, we can once more invoke the inequalities (3.12) or (3.30), respectively, to argue that those terms are of order $t^{-1}$. We can therefore rewrite eq. (3.36) as

$$
\lim _{t \rightarrow \infty}\left(\psi_{t}, H^{\nu} \psi_{t}\right)=\left\langle\chi, H_{1}^{\nu} \chi\right\rangle \text { for } \nu=0,1,2
$$

where, for $\nu=0$, we have just combined eqs. (2.26) and (3.31). Note that due to translational invariance in the variable $Z$, the expectation value on the rhs of eq. (3.37) is independent of $t$.

We are finally ready to prove the theorem stated in the introduction. Let $E \geqslant 0$ be an arbitrary energy value and $\epsilon>0$. We then choose a wave function $\chi$, as specified above, such that

$$
\langle\chi, \chi\rangle=1, \quad\left\langle\left(H_{1}-E\right) \chi,\left(H_{1}-E\right) \chi\right\rangle<\epsilon / 2
$$

It is easy to convince oneself that such functions $\chi$ always exist (take a plane wave and multiply it by a smooth slowly varying cutoff function of compact support). For this choice of $\chi$ and for sufficiently large $t$, the function $\hat{\psi}_{t}$ defined and discussed above is an element of $\hat{\mathscr{H}}$. Furthermore, the associated full wave function $\psi_{t}$ is differentiable, gauge invariant and satisfies eq. (3.37). It follows from this relation that

$$
\left|\left((H-E) \psi_{t},(H-E) \psi_{t}\right)-\left\langle\left(H_{1}-E\right) \chi,\left(H_{1}-E\right) \chi\right\rangle\right|<\epsilon / 2
$$

for (say) $t \geqslant T$. Consequently, for these values of $t$ we have

$$
\left\|\psi_{l}\right\|=1 \text { and }\left\|(H-E) \psi_{l}\right\|^{2}<\epsilon
$$

which proves the theorem (the second statement made in the theorem is a trivial consequence of the first and of the fact that $H \geqslant 0$ by supersymmetry, see eq. (2.12) and ref. [8], for example).

\section{Conclusions}

In this paper we have presented an investigation of a set of supersymmetric quantum mechanical models with a (global) gauge symmetry. These models are 
directly related to both the supersymmetric Yang-Mills theory and the supermembrane. Our basic mathematical result is that the Hamilton operator of these systems has a continuous spectrum starting at zero. The proof given is completely rigorous and, apart perhaps from the gauge fixing procedure, also rather simple.

Our primary motivation for this investigation was to analyze the spectrum of the eleven-dimensional supermembrane, and here our results do not bode well for its future. Although one might object that our results were derived only for finitedimensional gauge groups and therefore do not apply to the supermembrane proper, we are confident that our main conclusion will not be affected by taking the large $N$ limit. This is because the potential valleys which cause the instability are also present for infinite-dimensional groups (in fact, the subset of configurations with zero potential energy even becomes infinite-dimensional in this case), so that the basic physical picture embodied in the simple example of the introduction remains the same. Quite on the contrary, even if the spectrum had been discrete for finite $N$ (as is the case for the purely bosonic membrane) one would have been left with the problem of showing that it remains so in the limit of infinitely many degrees of freedom. However, we would still like to stress that the $N \rightarrow \infty$ limit is quite subtle and that we cannot a priori exclude a discontinuity at $N=\infty$, especially in view of the apparent non-renormalizability of the supermembrane as a three-dimensional field theory.

It is also worth emphasizing that the question of whether the supermembrane has massless states, i.e. whether there exists a set of normalizable eigenstates of $H$ with eigenvalue zero, remains open. (This question was addressed in refs. $[5,11]$ ). The relevance of this problem has, however, been greatly reduced by our results: massless states, if they occur, will not be separated from the continuum. In other words, the supermembrane describes a world without gap between low-energy and Planck-scale physics. It should also be clear that compactification of the supermembrane does not help since not all of its coordinates can be compactified if it is to describe the real four-dimensional world. We have not investigated whether these problematical features are absent for supermembranes moving in a non-trivial background.

As examples of supersymmetric quantum mechanics, the models discussed in this paper have been known for some time [12]. Our result shows that they are more intricate than expected. In particular, the possibility of a continuous spectrum has apparently not been envisaged so far. In these circumstances, the Witten index $\operatorname{Tr}(-1)^{F}$ is not a well-defined quantity anymore. Nevertheless, there have been attempts to calculate it through the path-integral representation of the partition function of the model, using the "ultralocal" approximation where the path integral is reduced to an ordinary integral [13]. In the light of our result, the significance of these calculations is not clear at present. Similar comments apply to the arguments presented in ref. [14].

Evidently, our investigation is also relevant for the understanding of supersymmetric gauge theories. At first sight, one might be worried that these field theories 
are subject to an instability, too, but as we shall now explain in some detail, the effect described in our paper actually has quite different implications here.

First, it is important to note that the operator (1.14) is obtained from the hamiltonian of the supersymmetric Yang-Mills theory by restricting the fields to be constant in space, and thus it is a truncated hamiltonian whose significance for field theory remains to be established. In the case of pure non-abelian gauge theories in four dimensions, it has been known for some time [6] that if one encloses the system in a finite (space-) box with periodic boundary conditions and linear extension $L$, the spectrum of the theory in the limit $L \rightarrow 0$ is exactly calculable. Furthermore, at low energies, it is given by the eigenvalues of the operator

$$
H^{\prime}=\frac{\bar{g}^{2 / 3}}{L}\left(\frac{1}{2} P_{a}^{A} P_{a}^{A}+\frac{1}{4} f_{A B E} f_{C D E} X_{a}^{A} X_{b}^{B} X_{a}^{C} X_{b}^{D}\right)
$$

where $\bar{g}$ denotes the "running" coupling at scale $L$. Thus, the reduction to constant gauge fields has a precise meaning in this theory. Note also that $H^{\prime}$ has a purely discrete spectrum [6,7], as appropriate for a theory in a finite volume.

Supersymmetric Yang-Mills gauge theories can also be formulated in a finite volume and one can then study the limit $L \rightarrow 0$. As a first guess, one would expect that the spectrum of the theory in this limit is obtained by dropping all non-constant modes, as in the bosonic case, so that one would end up with the Hamilton operator (1.14). However, as shown in this paper, the spectrum of this hamiltonian is continuous, and since this is rather unusual for a finite volume system, one is led to suspect that the small volume limit of supersymmetric Yang-Mills gauge theories is not given by the constant field model (1.14).

This conclusion is supported by the following considerations. At finite $L$ and in $D \leqslant 4$ spacetime dimensions, it is possible to calculate the spectrum of the full field theory hamiltonian in perturbation theory (this is because the effective coupling of the theory tends to zero in the limit $L \rightarrow 0$ ). To this end, one first determines the field configurations which minimize the bosonic potential and then expands about these minima. The potential is given by

$$
\int \mathrm{d}^{D-1} x F_{k l}^{A} F_{k l}^{A}
$$

where $F_{k l}^{A}$ denotes the gauge field strength and the indices $k, l$ run over the $D-1$ space directions. The absolute minima of this potential are obviously characterized by $F_{k l}^{A}=0$. Usually the solutions to these equations are just the pure gauge configurations, but on a torus, there are non-trivial solutions, referred to as "torons". It is easy to show that any toron is gauge equivalent to a constant gauge field taking values in a fixed Cartan subalgebra of the Lie algebra of the gauge 
group. Moreover, the manifold of all torons (modulo gauge transformations) is a compact orbifold.

When we now expand about the toron configurations, one finds that the groundstate wave function of the bosonic system is essentially supported in a small neighborhood of a set of singular points of the toron orbifold including the classical vacuum configuration [6]. The reason for this is that the potential (4.2) is not equally wide at all points of the toron orbifold in the directions transverse to it and consequently the wave function is suppressed at those points where the potential valley is narrow. In the analogous case of the bosonic operator $H_{\mathrm{B}}$ introduced in section 1 , the singular points of the toron orbifold correspond to the origin in the $x y$-plane (where the wave function prefers to live) while the lines $x=0$ and $y=0$ correspond to the non-singular parts of the orbifold.

In the supersymmetric case, the situation is completely different, because the zero-point energy associated with the fluctuations transversal to the toron orbifold is exactly cancelled by the contribution of the fermion degrees of freedom. Thus, there is no force driving the ground-state wave function to any particular points on the toron orbifold, and the spectrum of the model is hence given by a differential operator acting on functions defined on the toron orbifold. This, rather than the constant field hamiltonian (1.14) is the operator which describes the spectrum of the theory in the small volume limit, and since the toron orbifold is compact, it is clear that its spectrum will be discrete, as expected for a system in a finite volume.

The conclusion of this discussion is that the quantum mechanical system (1.14) is not actually the $L=0$ limit of the full supersymmetric Yang-Mills field theory, and the result of our paper therefore has no immediate impact on the latter. In particular, there is nothing which stands against the Witten index being a welldefined quantity in the field theory. The statement is only that $\operatorname{Tr}(-1)^{F}$ cannot be calculated by reducing to the constant field model as has been suggested by some authors.

We would like to thank U. Marquard and P. van Baal for discussions related to this work. B. de Wit is grateful to DESY for hospitality and financial support.

\section{References}

[1] E. Bergshoeff, E. Sezgin and P.K. Townsend, Phys Lett. B189 (1987) 75

[2] E. Bergshoeff, E. Sezgin and P.K. Townsend, Ann. Phys. 185 (1988) 330; P.K. Townsend, Cambridge preprint DAMPT-88-0732;

M. Duff, J. Class. Quant. Grav. 5 (1988) 189

[3] P.A.M. Dirac, Proc. R. Soc. A268 (1962) 57:

P.A. Collins and R.W. Tucker, Nucl. Phys. B112 (1976) 150;

P.S. Howe and R.W. Tucker, J. Phys, A10 (1977) L155;

A. Sugamoto, Nucl. Phys. B215 (1983) 381;

P. Gnadig, P. Hasenfratz, Z. Kunszt and J. Kuti, Ann. of Phys. 116 (1978) 380;

K. Kikkawa and M. Yamasaki, Progr. Theor. Phys. 76 (1986) 379 
[4] J. Goldstone, unpublished:

J. Hoppe, MIT PhD-Thesis, 1982: in Proc. Int. Workshop on Constraints theory and relativistic dynamics, eds. G. Longhi and L. Lusanna (World Scientific, Singapore, 1987)

[5] B. de Wit, J. Hoppe and H. Nicolai, Nucl. Phys. B305 [FS 23] (1988) 545

[6] M. Lüscher, Nucl. Phys. B219 (1983) 233

[7] B. Simon, Ann. Phys. 146 (1983) 209

[8] M. Reed and B. Simon, Methods of modern mathematical physics, Vols. 1-4 (Academic Press, New York, 1972)

[9] D.B. Fairlie, P. Fletcher and C.K. Zachos, preprint ANL-HEP-PR-88-56, DTP-88-13 (1988);

J. Hoppe, Karlsruhe preprint KA-THEP-18 (1988);

B. de Wit, U. Marguard and H. Nicolai, preprint DESY 89-031, THU-89-5 (1989)

[10] M.L. Metha, Random matrices (Academic Press, New York, 1967)

[11] M.J. Duff, T. Jnami, C.N. Pope, E. Sezgin and K.S. Stelle, Nucl. Phys. B297 (1988) 515;

L. Mezincescu, R. Nepomechie and P. Van Nieuwenhuizen, Nucl. Phys. B309 (1988) 317:

I. Bars, C.N. Pope and E. Sezgin, Phys. Lett. B198 (1987) 455

[12] M. Baake, P. Reinicke and V. Rittenberg, J. Math. Phys. 26 (1985) 1070:

R. Flume, Ann. Phys. 164 (1985) 189;

M. Claudson and M.B. Halpern, Nucl. Phys. B250 (1985) 689

[13] A.V. Smilga, Yad. Fiz. 42 (1985) 728; 43 (1986) 215; Nucl. Phys. B266 (1986) 45

[14] C.N. Pope and K.S. Stelle, J. Class. Quant. Grav. 5 (1988) L161 